\title{
O Uso de Famílias de Circuitos e Rede Neural Artificial para Previsão de Demanda de Energia Elétrica
}

\author{
José Leomar Todesco (Stela, INE - UFSC) tite@stela.ufsc.br \\ Francisco J. S. Pimentel (CELESC) franciscojsp@celesc.com.br \\ Arlan L. Bettiol (UNIVALI) bettiol@sj.univali.br
}

\section{Resumo}

A busca pela melhoria na qualidade da distribuição é uma constante nas empresas de energia. As metodologias atuais de supervisão das redes de distribuição utilizando geoprocessamento baseiam-se no cálculo de demanda dos transformadores através do uso de uma única curva de correlação $\mathrm{kWh} \times \mathrm{kVA}$ que emprega técnicas estatísticas. Este método é denominado método indireto de previsão da carga, e dele decorrem todos os cálculos elétricos do circuito para aferir seu desempenho quanto à qualidade dos níveis de tensão, do carregamento dos equipamentos e condutores, do balanceamento da carga, bem como das perdas elétricas. Este artigo apresenta uma proposta de estudo das famílias de circuitos (residencial, comercial, industrial, rural, público e outros) utilizando informações sobre o consumo de energia através da aplicação de algoritmos de clustering e, posteriormente, da aplicação de uma Rede Neural Artificial (RNA) para a aproximação das curvas de demanda para as famílias identificadas. A RNA utilizada foi a Radial Basis Function (RBF), por ser um aproximador universal. São também apresentados no artigo testes comparativos realizados com o modelo de regressão linear simples e a RBF.

Palavras-chave - Previsão de carga, Rede neural artificial, Estimativa da curva de demanda.

\section{Introdução}

Um dos objetivos principais de uma empresa de energia elétrica é oferecer um serviço de alta qualidade a seus consumidores, garantindo-lhes uma tarifa razoável a partir da melhor gestão da rede elétrica de distribuição. Para tal, deve procurar alocar de forma otimizada os recursos operacionais e de investimentos na rede de distribuição. A busca pela melhoria na qualidade da distribuição é uma constante nas empresas de energia. As metodologias atuais de supervisão das redes de distribuição que empregam geoprocessamento baseiam-se no cálculo de demanda dos transformadores, realizado através do uso de uma única curva de correlação $\mathrm{kWh} \times \mathrm{kVA}$ que utiliza técnicas estatísticas. Esse método é denominado método indireto de previsão da carga, e dele decorrem vários cálculos elétricos do circuito para aferir seu desempenho quanto à qualidade dos níveis de tensão, do carregamento dos equipamentos e condutores, do balanceamento da carga, bem como das perdas elétricas.

Tal gestão é feita a partir do conhecimento da situação operacional de suas redes, a qual é determinada pelo fluxo de corrente nos condutores e no carregamento elétrico de seus transformadores tanto nas condições normais de trabalho quanto nas situações de emergência. A metodologia mais tradicional de modelagem das cargas de consumidores consiste em considerar, para um dado transformador, o somatório dos consumos dos clientes, sem levar em consideração as particularidades de cada grupo de consumidores (residenciais, comerciais, industriais, rurais, públicos e outros). Além disso, para a estimativa da curva de correlação kWh x kVA, geralmente é utilizada a regressão linear simples, o que torna o modelo pouco preciso para a gestão de distribuição elétrica. 
Este artigo apresenta os resultados da aplicação de técnicas de IA [1] para o estudo das famílias de circuitos (residencial, comercial, industrial, rural, público e outros), utilizando informações sobre o consumo de energia através da aplicação de algoritmos de clustering e, posteriormente, da aplicação de uma Rede Neural Artificial (RNA) para a aproximação das curvas de demanda para as famílias identificadas.

O artigo está dividido em 6 seções, como se segue: seção I Introdução, seção II Gerência de Redes de Baixa Tensão (BT), seção III Métodos Empregados, seção IV Resultados, seção V Conclusões, seção VI Referências Bibliográficas.

\section{Gerência de Redes de BT}

Em face da crescente competitividade e dos critérios de qualidade e de formulação de preço impostos pela agência de regulação do setor elétrico (ANEEL), as empresas de distribuição de energia elétrica no Brasil são forçadas a buscar alternativas para a melhoria do processo de gestão dos circuitos de alta e baixa tensão.

Para o gerenciamento eficiente da rede de distribuição de energia, devem ser consideradas as particularidades de cada um dos vários circuitos secundários, baseados nas peculiaridades dos muitos consumidores do respectivo circuito. $\mathrm{O}$ conhecimento das várias famílias de circuitos, agrupadas por parâmetros técnicos, tais como indicadores de confiabilidade, níveis de carregamento, níveis de tensão, de balanceamento das cargas, energia comercializada mensalmente, e também por parâmetros não técnicos, como o perfil dos consumidores com as suas variadas características e diversidades, não é tarefa das mais fáceis. Nas Centrais Elétricas de Santa Catarina (CELESC), os parâmetros técnicos e não técnicos estão armazenados nos sistemas computacionais da empresa e guardam históricos de maneira que seja possível aplicar técnicas de agrupamento e conhecer as famílias de circuitos.

As metodologias atuais de gestão de energia utilizam o cálculo de demanda (KVA) de forma indireta, ou seja, baseiam-se no consumo de energia elétrica (KWh) mensal dos consumidores ligados aos transformadores, estando a eles associadas pela função de correlação $\mathrm{KWh} \times$ KVA [7]. cada ciclo do faturamento tem-se nova estimativa de demanda do circuito, e dos cálculos elétricos decorrentes estabelece-se o processo de gestão da rede de distribuição.

Este processo indireto é utilizado para a telesupervisão do sistema de distribuição e dos circuitos secundários, mas, ainda não se mostrou viável economicamente. Para supervisionar os circuitos secundários através de medições diretas seria necessário modificar os atuais medidores de energia em cada consumidor e instalar sistemas de teleleitura em cada circuito transmitindo em ondas portadoras (ex.: VHF) as informações das grandezas elétricas medidas no transformador, com varreduras periódicas durante o dia, supervisionadas por sistemas computacionais para possibilitar o controle dos circuitos. Esta tecnologia já existe, mas ainda é muito cara.

A configuração da rede de distribuição pode ser bem determinada através de sistemas de cadastros mantidos nos sistemas operacionais da empresa e que são atualizados freqüentemente por visitas em campo através do acompanhamento das novas obras. Já o comportamento dos consumidores é altamente variável e diversificado.

Tal diversidade de comportamento introduz uma grande dificuldade na determinação correta do estado operacional de um circuito secundário de distribuição, do ponto de vista dos valores calculados pelos algoritmos de cálculo elétrico. $\mathrm{O}$ cálculo de demanda dos transformadores hoje é realizado pelo uso de curvas de correlação $\mathrm{kWh} \times \mathrm{kVA}$. A maioria das metodologias atuais utiliza a regressão linear simples para estimar as curvas de correlação $\mathrm{kWh} \times \mathrm{kVA}$. Recentemente, um estudo foi feito na COPEL utilizando a conversão Consumo/Demanda [7], tendo sido utilizada uma função geométrica para a estimativa. 
A Figura 1 a seguir apresenta um exemplo de curva de correlação "ajustada" a um conjunto de medições hipotético.

A possibilidade da aplicação de algoritmos de reconhecimento de padrões [9] [3] [4], especificamente os algoritmos de clustering, é uma alternativa viável para a caracterização de agrupamentos de famílias de circuitos, baseados em parâmetros, técnicas e dados dos consumidores.

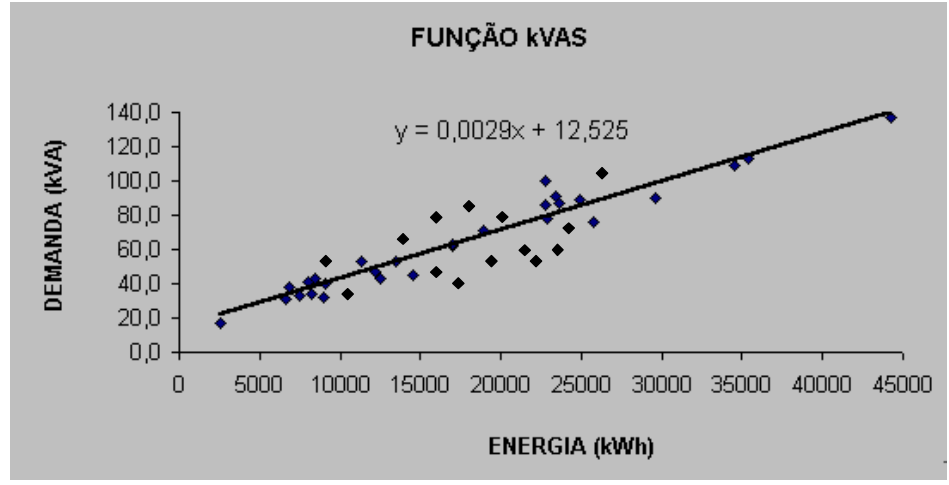

Figura 1 - Curva de correlação kWh x kVA

Uma vez identificados os agrupamentos e determinadas as diferentes famílias de circuitos, há a possibilidade de se estimar a demanda desses circuitos através do uso da aplicação de redes neurais artificiais [5] [4], em substituição às curvas de correlação (KWh x KVA) atualmente utilizadas.

A metodologia neste projeto propõe que novas curvas de correlações sejam determinadas a partir da aplicação de redes neurais artificiais para cada uma das famílias identificadas no agrupamento de circuitos. A seguir, são apresentados os principais métodos empregados.

\section{Métodos Empregados}

Para o agrupamento das famílias, foi utilizado o algoritmo de clustering k-means. Os algoritmos de clustering buscam nos dados estruturas de agrupamentos naturais de maneira que a identificação desses agrupamentos possa sugerir hipóteses interessantes de relacionamentos [9] [2][8].

\subsection{Algoritmos de Clustering}

O algoritmo k-means baseia-se na minimização de uma medida de custo, na distância interna entre os padrões de um agrupamento. A minimização do custo garante encontrar um mínimo local da função objetivo, que dependerá do ponto inicial do algoritmo. Esse tipo de algoritmo é chamado de "não-convexo", pois, a cada iteração diminui o valor da distorção, visto que o resultado final depende do ponto inicial usado pelo algoritmo. Uma derivação do algoritmo kmeans, com heurísticas para divisão e união de "clusters", é o algoritmo ISODATA, que é o algoritmo k-means modificado. Apesar dessas características, o ISODATA também não é convexo e variante, ou seja, enfrenta problemas quando se depara com mínimos locais durante a minimização do custo[1]. Observe-se que: " dado um conjunto $\mathrm{S}$, distorção é qualquer

aplicação d: S X S $\rightarrow{ }^{\mathfrak{R}_{0}^{+}}$que associa cada par de elemento de $\mathrm{S}$ a um número real que traduz o afastamento entre esses elementos" [21].

\section{$\mathrm{O}$ algoritmo $k$-means}

I. Inicialização: Selecionar arbitrariamente os $\mathrm{k}$ centros iniciais de agrupamentos (centróides), Z1, Z2,..., Zk.

II. Determinar a distância , com i $=1,2, \ldots, k$, entre cada centróide e os demais padrões. A designação dos padrões às classes de padrão se fará, no caso da distância euclidiana, quando as amostras de padrão atenderem à relação:

$$
\left\|x_{i}-Z_{j}(k)\right\|<\left\|x_{i}-Z_{i}(k)\right\|, \text { com } \mathrm{i}=1,2, \ldots, \mathrm{k} \text { e } \mathrm{i} \neq \mathrm{j} \text {. }
$$


A expressão $\left\|x_{i}-Z_{j}\right\|$ define a distância euclidiana entre os padrões (amostras) e os centróides. Formam-se $\mathrm{k}$ agrupamentos (cluster), compostos dos elementos $\mathrm{X}_{\mathrm{i}}$ pertencentes à classe $S_{j}(k)=\left\{x_{1}, x_{2} \ldots\right\}$.

III. Atualizar os centros de agrupamento a partir do passo 2, usando a relação:

$Z_{j}(k+1)=\frac{1}{N} \sum_{x \in S_{j}} x$, onde $\mathrm{N}$ é número de elementos de $\mathrm{S}_{\mathrm{j}}$. Esta atualização minimiza a soma do quadrado das distâncias de cada padrão do agrupamento ao novo centro de agrupamento.

IV. Comparar os centróides com o $Z_{j}(k+1)$ com o centróide $Z_{j}(k)$. Se $Z_{j}(k+1) \neq Z_{j}(k)$ então novos centróides ficam determinados e repete-se o passo $2 \mathrm{com}_{Z_{j}(k+1)}$ no lugar de $Z_{j}(k)$, definindo assim os novos de cada classe. Se o algoritmo converge e o procedimento está terminado, com a designação de cada padrão à sua respectiva classe de padrões.

$\mathrm{O}$ comportamento do algoritmo k-means apresenta vantagens no que concerne à simplicidade e à eficiência. É rápido para cálculos simples, possibilitando o processamento seqüencial dos dados, acarretando baixa quantidade de armazenamento de informações a serem processadas. A desvantagem é a sua dependência dos valores iniciais de $\mathrm{k}$, da ordem em que as amostras são processadas, da escolha dos primeiros centros de agrupamento e da geometria das amostras disponíveis para análise. Em alguns casos sua utilização requer experimentação com vários valores de $\mathrm{k}$ e diferentes escolhas dos parâmetros iniciais [4].

\subsection{Redes Neurais Artificiais}

O interesse em redes neurais data do início da década de 40, com o trabalho pioneiro de McCulloch e Pitts [6]. Warren McCulloch foi um psiquiatra e neuroanatomista por treinamento que estudou por 20 anos a representação de um evento no sistema nervoso. Walter Pitts foi um prodígio matemático que se uniu a McCulloch em 1942, para em 1943 escreverem um artigo que se tornou clássico e recebeu muita atenção da comunidade que estudava o modelo do neurônio [6].

Broomhead e Lowe em 1988 estão entre os primeiros a explorar o uso da rede neural com função de base radial (RBF) no projeto de RNA [6], com contribuições de Moody e Darken. Renal em 1989 e Poggio e Girosi também trouxeram contribuições para a RBF. A construção de uma RBF em sua forma mais básica envolve três camadas, cujos nodos de saída formam uma combinação linear das funções de base radial (kernel) calculados pelos nodos da camada escondida. As funções de base radial na camada escondida produzem uma resposta localizada para o estímulo (padrão) de entrada, isto é, produzem uma resposta significativamente diferente de zero somente quando o padrão de entrada está dentro de uma região pequena localizada no espaço de entradas. RBF usando não-linearidade local com decréscimo exponencial, como é o caso da função de Gauss, constrói aproximações locais para mapas de entrada/saída não lineares. Nesse sentido, as RBFs são capazes de aprender mais rápido e têm sensibilidade reduzida com respeito à ordem de apresentação dos dados de treinamento.

Dado um vetor de entrada $\mathrm{x}$, a saída de um nodo simples será $\mathrm{y}=f(\mathrm{x}-\mathrm{c})$ onde, por exemplo, a função $f$ pode ser tomada como $f=(\mathrm{x}-\mathrm{c})=\frac{1}{(2 \pi)^{n / 2} \sigma_{1} \cdot \sigma_{2} \ldots \sigma_{n}} \exp \left\{-\frac{1}{2} \sum_{j=1}^{n}\left(\frac{\boldsymbol{x}_{j}-\boldsymbol{c}_{j}}{\sigma_{j}}\right)^{2}\right\}$

Os valores $\sigma_{1} \cdot \sigma_{2} \ldots \sigma_{n}, \mathrm{j}=[1, \mathrm{n}]$ são usados da mesma maneira que na distribuição de probabilidade normal para determinar a dispersão escalar em cada direção. Uma outra variação comum nas funções bases é aumentar sua funcionalidade usando a distância de Mahalanobis na função Gaussiana. A equação anterior torna-se: $\boldsymbol{f}=(\mathrm{x}-\mathrm{c})=\frac{1}{(2 \pi)^{n / 2}|\boldsymbol{K}|^{1 / 2}} \exp \left\{-\frac{1}{2}(\boldsymbol{x}-\boldsymbol{c})^{T} \boldsymbol{K}^{-1}(\boldsymbol{x}-\boldsymbol{c})\right\}$

onde K-1 é a inversa da matriz co-variância de X 
associada com o nodo da camada escondida C.

Dados n-vetores (dados de entrada) de p-exemplares, representando p-classes, a rede pode ser iniciada com conhecimento dos centros (localizações dos exemplares). $\mathrm{Se}^{\mathrm{c}_{j}}$ representa o jésimo vetor exemplar, então pode-se definir a matriz peso $\mathrm{C}$ como: $\mathrm{C}=\left[\mathrm{c}_{1} \mathrm{c}_{2} \ldots \mathrm{c}_{3}\right]^{\mathrm{T}}$ tal que os pesos na camada escondida do nodo j são compostos do vetor "centro" $\mathrm{c}_{j}$.

A camada de saída é uma soma ponderada das saídas da camada escondida. Quando apresentado um vetor de entrada $\mathrm{x}$ para rede, a rede implementa $\mathrm{y}=\mathrm{W} \cdot f(\|\mathrm{x}-\mathrm{c}\|)$

onde $f$ representa o vetor de saída funcional da camada escondida, e C o correspondente vetor centro. Fornecido alguns dados com respostas desejadas, os pesos $\mathrm{W}$ podem ser determinados usando-se o algoritmo de treinamento LMS interativamente ou nãointerativamente, como técnicas do gradiente descendente e pseudo inversa, respectivamente.

O Aprendizado na camada intermediária é executado usando-se um método não supervisionado, tipicamente um algoritmo de "cluster", um algoritmo de cluster heurístico, ou um algoritmo supervisionado para achar os centros (nodos $\mathrm{C}$ na camada escondida). $\mathrm{O}$ algoritmo mais comum usado para determinar os centros (que são as conexões entre a camada de entrada e a camada intermediária) é o algoritmo K-means [4], apresentado anteriormente.

Em alguns estudos também foram utilizados aprendizado supervisionado das localizações dos centros e aprendizado auto-organizativo dos centros, como apresentados no trabalho de Todesco [9], ou ainda o algoritmo dos mínimos quadrados ortogonais (OLS) desenvolvido por Chen, Cowan e Grant em 1990 [9].

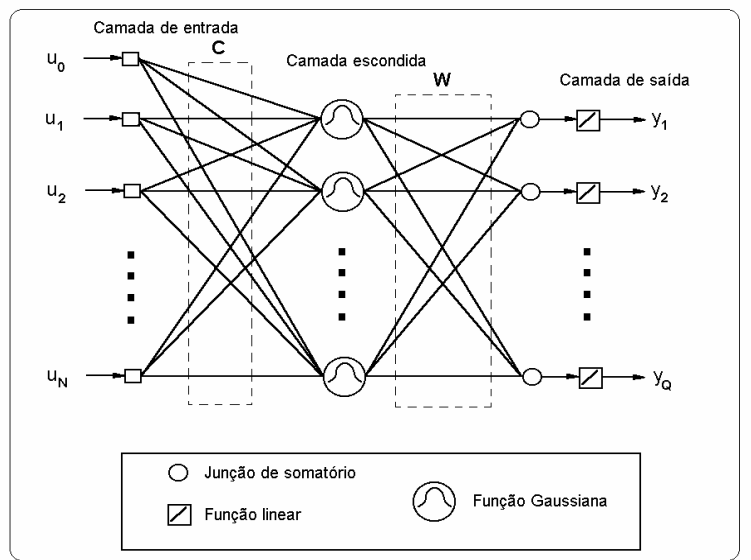

Figura 2 - Estrutura Básica da $R B F[6]$

Uma forma simples para determinar o parâmetro de variação $\sigma^{2}$ para as funções de Gauss é colocá-las igual à distância média entre todos os dados de treinamento $\sigma_{j}^{2}=\frac{1}{M_{j}} \sum_{x \in \Theta_{j}}(x-c)^{T}(x-c)$

onde ${ }^{\Theta}{ }_{j}$ é o conjunto de padrões de treinamento agrupados no centro do cluster $\mathrm{Cj}$, e Mj é o número de padrões em ${ }^{\Theta_{j}}$.

Uma outra maneira de escolher o parâmetro $\sigma^{2}$ é calcular as distâncias entre os centros em cada dimensão e usar alguma porcentagem dessa distância para o fator de escala. Nesse sentido, o algoritmo p-nearest nerghbor tem sido usado.

\section{Resultados}

\subsection{Classificação das Famílias}

Os atributos utilizados para a classificação foram as variáveis disponíveis na organização em 
seus sistemas operativos. Para a determinação das famílias, foi utilizada uma amostragem de 22.376 circuitos secundários das regionais de Florianópolis, Joinville, Blumenau e Tubarão.

Várias análises estatísticas sobre esses atributos (variáveis) foram realizados para um melhor entendimento da amostra. Posteriormente, as variáveis foram padronizadas utilizando-se o consumo percentual por tipo, ou seja, \%KWH_RES. Desta forma, houve uma normalização desses dados. Após várias análises, observou-se que os dados que mais contribuíam para o agrupamento foram \%KWHRES, \%KWHCOM, \%KWHIND, \%KWHRUR, \%KWHPUB e $\%$ KWHOUT.

Para os testes, ou seja, circuitos com medições em campo da demanda de energia, foram disponibilizadas 429 observações.

Primeiramente, os circuitos foram separados em duas grandes classes $\mathrm{C} 1$ e C2, ou seja, C1 é a classe mista e C2 é a classe residencial. Para essa separação foi aplicado o algoritmo k-means. O Quadro 1 a seguir mostra os valores encontrados para o centro do cluster e os desvios para cada um dos tipos de consumo.

Posteriormente, para cada uma das classes, novamente foram realizados estudos $\mathrm{e}$ encontraram-se cinco novos agrupamentos para a Classe C1 (Nres). Esses cinco agrupamentos foram denominados famílias não residenciais. $\mathrm{O}$ mesmo ocorreu para $\mathrm{C} 2$, em que foram encontradas cinco novas famílias, denominadas residenciais.

\begin{tabular}{|c|c|c|}
\hline \multicolumn{3}{|c|}{ Quadro 1 - Classes C1 e C2 } \\
\hline Média & C1 (NRes) & C2(Res) \\
\hline \%KWHRES & 13,00 & 88,10 \\
\hline \%KWHCOM & 40,22 & 5,71 \\
\hline \%KWHIND & 21,39 & 3,64 \\
\hline \%KWHPUB & 5,47 & 0,71 \\
\hline \%KWHRUR & 18,14 & 1,69 \\
\hline \%KWHOUT & 1,78 & 0,15 \\
\hline
\end{tabular}

Para a classificação de um circuito qualquer em uma das famílias é realizado o cálculo da distância euclidiana, e será o circuito atribuído a classe que obtiver a menor distância.

\subsection{Aproximação com a RBF}

Assim, para o experimento com a rede neural, foi utilizada a base de testes com uma amostra de 429 medições, que, após eliminação de alguns ruídos, permaneceu com 427 medições de circuitos disponíveis no sistema ANAWIN. Este conjunto se mostrou insuficiente para treinar uma rede para cada uma das 10 famílias de circuitos. Portanto, primeiramente foram realizados estudos sobre as duas classes, ou seja, residencial e não residencial.

A classe residencial contou com 331 medições e a não residencial, com 96 medições. Após separar os dois conjuntos (residencial e não residencial), separou-se novamente cada uma das sub-mostras em conjunto de treinamento da rede ( $2 / 3$ da sub-mostra) e conjunto de teste $(1 / 3$ da sub-mostra).

Para a classe residencial, foram utilizadas seis entradas, após vários experimentos, as entradas foram o número médio de consumidores residenciais, KWHTOTAL, KWHRES, KWHCOM, KWHIND e KWHRUR. O conjunto de treinamento contou com 221 observações, e o de testes, com 110. O melhor resultado obtido foi com o seguinte experimento 1.

A Figura 3 a seguir mostra o gráfico do resultado da aproximação da RBF com as medições da classe residencial

Para a classe não residencial, foram utilizadas quatro entradas, ou seja, a média do KWHRES, KWHCOM, KWHIND e KWHRUR. Para o treinamento foram utilizadas 64 medições, e para os testes, 32. O melhor resultado foi encontrado com o experimento 2. 


\section{Experimento 1}

Número de centros $=15$

Fator do raio $=8$

Erro Médio $=8,3123$

Erro Quadrado = 12,2967

\section{Experimento 2}

Número de centros $=15$

Fator do raio $=6$

Erro Médio $=12,5336$

Erro Quadrado = 20,8531

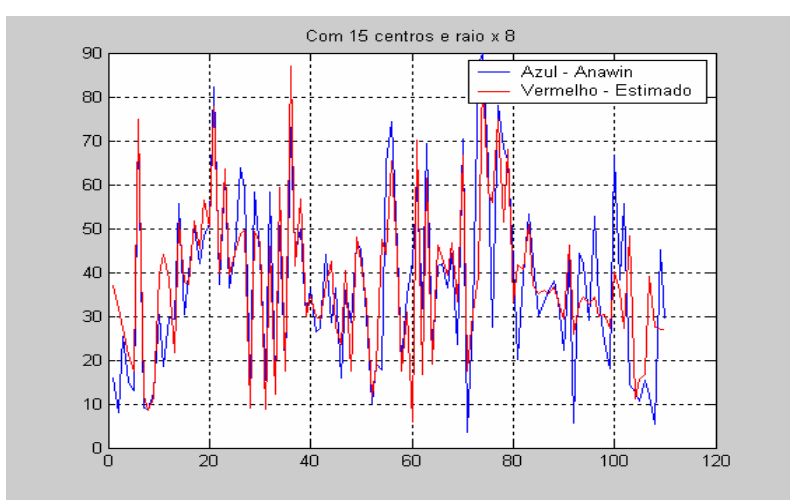

Figura 3 - Aproximação da RBF para a classe residencial

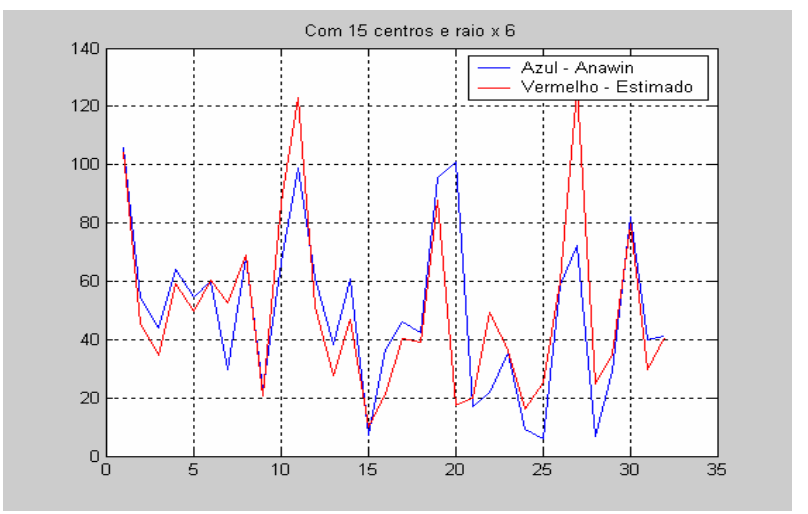

Figura 4 - Aproximação da RBF para a classe não residencial

Já para a classe não residencial, o erro foi maior, conforme já esperado. Vale também lembrar que no modelo de regressão não existe separação entre dados de treinamento e dados de testes, sendo todos os dados são utilizados para a minimização dos erros no ajuste da curva, diferentemente da rede neural.

A Figura 4 a seguir mostra o gráfico do resultado da aproximação da RBF com as medições da classe não residencial.

O Quadro 2 a seguir apresenta (a) o resultado da aplicação do modelo adotado pela CELESC para a estimação da demanda a partir do $\mathrm{kWh}$ e (b) a aplicação da regressão linear simples e múltipla para todos os dados, após classificação em $\mathrm{C} 1$ e C2 e nas 10 famílias, através da taxa de erro absoluto.

Conforme pode ser observado, tanto para a regressão simples (RS) quanto para a regressão múltipla (RM) foi possível aplicar o modelo para as dez famílias. Na última linha, denominada geral, mostra-se o erro médio da classificação para a técnica.

Comparando-se o resultado da RBF para a classe não residencial $(\mathrm{C} 1)$ que obteve uma taxa de erro de $12,53 \%$, conforme já mostrado anteriormente, esse resultado está abaixo apenas da aplicação da regressão linear múltipla para as cinco famílias dessa classe. Mas se comparado com o uso somente para as classes $\mathrm{C} 1$, já se verifica que o resultado da RBF é melhor.

Quadro 2 - Resultados

\begin{tabular}{|l|l|l|l|l|}
\hline CELESC & RS-C1C2 & RM-C1C2 & RS-Fam & RM-Fam \\
\hline
\end{tabular}

\begin{tabular}{|c|c|c|c|c|c|c|}
\hline & NRes 1 & 16.18 & 18.47 & 17.46 & 16.55 & 14.09 \\
\hline & NRes 2 & 4.21 & 6.35 & 4.54 & 4.30 & 3.66 \\
\hline $\mathrm{C} 1$ & NRes 3 & 19.83 & 20.11 & 19.97 & 20.45 & 19.29 \\
\hline (NRes) & NRes 4 & 21.58 & 19.97 & 19.81 & 11.97 & 0.00 \\
\hline & NRes 5 & 11.16 & 10.26 & 10.48 & 8.89 & 6.22 \\
\hline & C1 & 13.08 & 13.27 & 13.25 & 12.64 & 11.59 \\
\hline & Res_1 & 13.88 & 14.15 & 14.14 & 13.88 & 12.75 \\
\hline & Res_2 & 8.35 & 8.35 & 8.28 & 7.58 & 7.57 \\
\hline $\mathrm{C} 2$ & Res_3 & 8.05 & 8.64 & 8.70 & 7.91 & 7.62 \\
\hline (Res) & Res_4 & 7.54 & 8.30 & 8.50 & 6.39 & 5.70 \\
\hline & Res_5 & 9.86 & 10.72 & 10.51 & 7.28 & 4.36 \\
\hline & C2 & 9.49 & 9.80 & 9.80 & 8.96 & 8.49 \\
\hline & eral & 10.31 & 10.58 & 10.58 & 9.79 & 9.19 \\
\hline
\end{tabular}


Para a classe residencial, com o uso da RBF obteve-se uma taxa de erro de $8,31 \%$ que foi melhor do que todos os outros métodos, diminuindo em uma unidade o valor da taxa de erro se comparado à classe $\mathrm{C} 2$.

Se fizermos o erro médio geral, ou seja, a média ponderada da taxa de erro da classe residencial e não residencial, lembrando que os circuitos residenciais são os predominantes, para o uso da RBF teremos uma taxa média de 9,25\%. Comparando essa taxa média com as taxas médias dos modelos de regressão, a aproximação está muito próxima da regressão linear múltipla para as dez famílias analisadas.

Acredita-se que, a partir do aumento da amostra de medições, será possível treinar uma rede para cada uma das dez famílias, e assim, melhorar a taxa de erro consideravelmente, sendo possível diminuí-la ainda mais a taxa de erro e ultrapassar o resultado da regressão linear múltipla.

\section{Conclusão}

Neste artigo uma RBF foi proposta para aproximação de curvas de demanda de energia para circuitos de baixa tensão.

Foram utilizadas 427 medições de circuitos de baixa tensão, que após aplicação do algoritmo k-means foram separados em duas grandes famílias (residencial e não residencial) e para cada uma dessas famílias foi treinada uma rede neural RBF. Para a classe residencial a taxa de erro encontrada foi de $8,31 \%$, taxa muito boa quando comparada aos modelos de regressão linear simples e múltipla. Para a classe não residencial, a taxa de erro foi de $12,53 \%$, também muito boa quando comparada aos outros modelos.

Atualmente o conjunto de RBFs está sendo melhorado a partir do aumento do conjunto de medições, o que possibilitará a aproximação de uma RBF para cada uma das dez famílias determinadas para os circuitos.

\section{Referências Bibliográficas}

[1] DABBAGHCHI, I. et al. AI Application Areas in Power Systems. IEEE Expert, v. 12, n. 1, p. 58-66, 1997.

[2] BETTIOL, Arlan Luiz et al. Estimation of Critical Clearing Times Using Neural Networks. IEEE Proceedings On Electric Power Applications, Bologna, Italia, v. 1, n. 1, p. 392-398, 2003.

[3] PAO, Yoh-Han. Adaptative Pattern Recognition and Neural Networks. Addison-Wesley Publishing Co, Inc., 1989.

[4] DUDA, R. O. \& HART. Pattern Classification and Scene Analysis. John Wiley \& Sons, 1973.

[5] BISHOP, R. Pattern Recognition and Neural Networks. Addison \&Wesley, 1996.

[6] HAYKIN, S. Neural Networks: A Comprehensive Foundation. New York: Macmillan College Publishing Company, 1994

[7] SILVA, M. D. L. Conservação Consumo/Demanda: Uma Metodologia Alternativa. Relatórios técnicos da COPEL, PR, 2001.

[8] TODESCO, J. L. et al. Transient Stability Analysis of Power Systems Using Neural Networks. In: VIII SEPOPE, 2001, São Carlos - SP. VIII SEPOPE. 2001.

[9] TODESCO, J. L. Reconhecimento de Padrões Usando Rede Neuronal Artificial com uma Função de Base Radial: Uma Aplicação na Classificação de Cromossomos Humanos. Tese (Doutorado em Engenharia de Produção) - Engenharia de Produção e Sistemas, UFSC. Florianópolis, 1995. Orientador: Edgar Augusto Lanzer. 